\title{
Imaging the Surface of a Star: VLBA Monitoring of the SiO Masers around U Herculis
}

\author{
P. J. Diamond \& A. J. Kemball \\ National Radio Astronomy Observatory, Socorro, NM 87801, USA
}

Abstract. We report on 4 epochs of VLBA polarization observations of the $43 \mathrm{GHz}, \mathrm{v}=1$, $\mathrm{J}=1-0 \mathrm{SiO}$ masers in the circumstellar envelope around the Mira variable U Herculis.

\section{Total Intensity Structure}

VLBA observations of $43 \mathrm{GHz} v=1, \mathrm{~J}=1-0 \mathrm{SiO}$ masers around stars have revealed ring-like structures in almost every object studied. The masers reside just above, the photospheres of the stars. Monitoring shows dramatic structural changes on timescales of months.

$\mathrm{U}$ Herculis is a fairly typical Mira variable star. It lies at a distance of about $460 \mathrm{pc}$, has a period of $\sim 405$ days. It exhibits strong $\mathrm{SiO}$ maser emission from several vibrational and rotational transitions. It also contains strong $\mathrm{OH}$ and $\mathrm{H}_{2} \mathrm{O}$ masers. Early VLBA observations (May 1992) showed that the masers lay in a narrow elongated ring with a north-south dimension of 21 mas $(\approx 9.7 \mathrm{AU})$ and an east-west dimension of 16 mas $(\approx 7.4 \mathrm{AU})$. The mean FWZP of the ring at that time was 2.6 mas $(\approx 1.2 \mathrm{AU})$ (Diamond et al. 1994).

Table 1. Major axes of SiO ring of U Herculis.

\begin{tabular}{|c|c|c|c|}
\hline Date & $\begin{array}{c}\text { E-W diam. } \\
\text { AU }\end{array}$ & $\begin{array}{c}\text { N-S diam. } \\
\text { AU }\end{array}$ & $\begin{array}{c}\text { FWZP of ring } \\
\text { AU }\end{array}$ \\
\hline 30 June 1995 & 10.6 & 12.2 & 1.2 \\
29 Dec 1995 & 11.8 & 11.8 & 1.9 \\
2 Feb 1995 & 10.6 & 12.0 & 1.1 \\
5 Apr 1996 & 10.7 & 10.7 & 1.1 \\
\hline
\end{tabular}

Fig. 1 shows the full polarization images of $U$ Herculis made with the VLBA between June 1995 and April 1996. From these and the earlier data we can see that the ring nature of the maser emission is consistent throughout 3.6 stellar cycles. However considerable detailed changes occur on timescales as short as one month.

Measurements of the major and minor axes of the ring-like structure are given in Table 1. Changes of up to $1.5 \mathrm{AU}$ are visible. We do not however see the clear signature of contraction visible in similar observations of $\mathrm{R}$ Aqr (Boboltz et al. 1997).

\section{Polarization Structure}

Kemball \& Diamond (1997) have shown that in the star TX Cam the orientation of the electric vectors is predominately tangential to the ring. This is interpreted as being caused by a poloidal magnetic field confined to the surface of the extended atmosphere. 

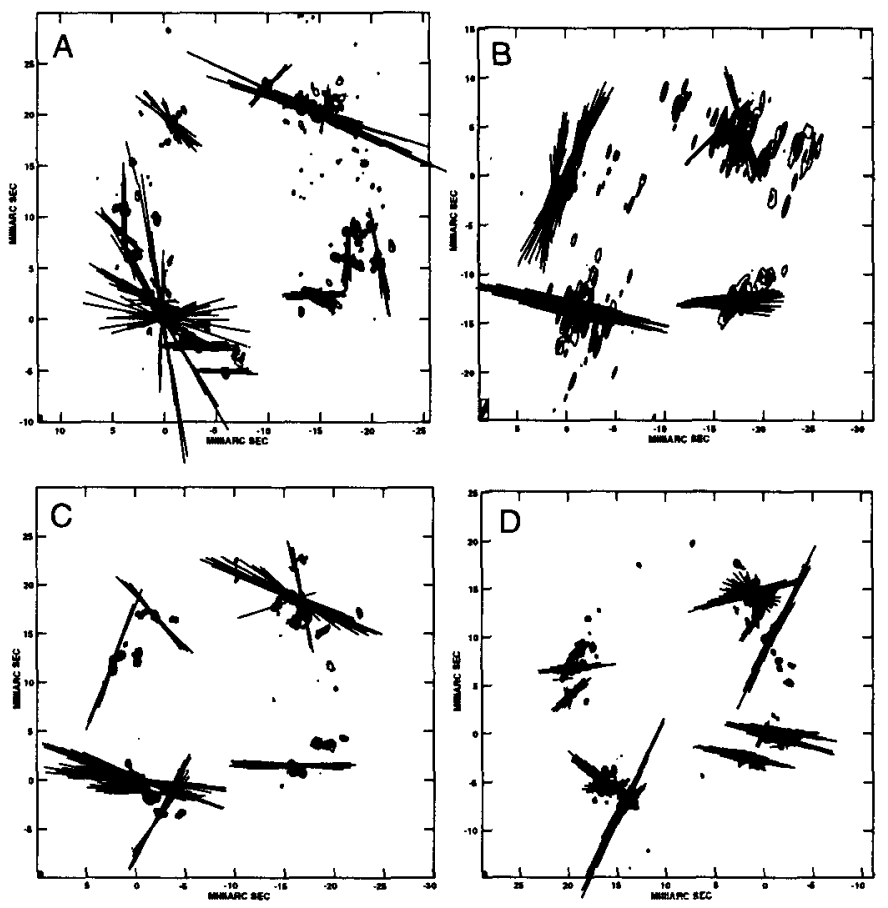

Figure 1. 4 images of the total intensity and linearly polarized electric vectors of the $43 \mathrm{GHz} \mathrm{SiO}$ maser emission from U Her. The images show the emission integrated over the velocity range of the maser: a) June 1995; b) Dec 1995; c) Feb 1995; d) Apr 1995.

Figure 1 demonstrates that the clear tangential linear polarization structure seen in TX Cam is not as evident in U Her. The position angles of the vectors do show some consistency from epoch to epoch and tangential vectors are present (statistical analysis to come in a future paper).

The degree of circular polarization $m_{c}$ is seen to change on very short timescales, $<1$ month. The mean $m_{c}$ varies from $<1 \%$ in Dec 1995 to $\approx 3-5 \%$ in April 1996. Based on the expression for $B \sec \theta$ from Kemball \& Diamond (1997) this implies a mean magnetic field that varies from $\approx 1 \rightarrow 5$ Gauss. Alternatively the magnetic field strength may remain approximately constant and the angle $\theta$ between the line of sight and the magnetic field direction may vary. More detailed analysis of the data is necessary to determine the true picture.

Acknowledgments. The National Radio Astronomy Observatory is a facility of the National Science Foundation, operated under a cooperative agreement by Associated Universities, Inc.

\section{References}

Boboltz, D. A., et al. 1997. $A p J, 487$ L147-150.

Diamond, P. J., et al. 1994. $A p J, 430$, L61-65.

Kemball, A. J. \& Diamond, P. J. 1997. $A p J$, 481, L111-114. 